\title{
On the Widespread Occurrence of the Inverse Square Distribution in Social Sciences and Taxonomy
}

\author{
Guido Caldarelli, ${ }^{1,2}$ Cécile Caretta Cartozo, ${ }^{1}$ Paolo De Los Rios, ${ }^{3}$ and Vito D.P. Servedio ${ }^{1}$ \\ ${ }^{1}$ INFM UdR ROMA1 Dipartimento di Fisica, Università di Roma "La Sapienza" Piazzale A. Moro 200185 Roma, Italy \\ ${ }^{2}$ Istituto di Studi e Ricerche "E. Fermi" Compendio Viminale 00184 Roma, Italy \\ ${ }^{3}$ Laboratoire de Biophysique Statistique, ITP-FSB, \\ Ecole Polytechnique Fédérale de Lausanne, CH-1015 Lausanne, Switzerland
}

(Dated: November 21, 2018)

\begin{abstract}
The widespread occurrence of an inverse square relation in the hierarchical distribution of subcommunities within communities (or sub-species within species) has been recently invoked as a signature of hierarchical self-organization within social and ecological systems. Here we show that, whether such systems are self-organized or not, this behavior is the consequence of the tree-like classification method. Different tree-like classifications (both of real and truly random systems) display a similar statistical behaviour when considering the sizes of their sub-branches.
\end{abstract}

PACS numbers: $05.40+\mathrm{j}, 64.60 \mathrm{Ak}, 64.60 \mathrm{Fr}, 87.10+\mathrm{e}$

Taxonomy is one of the major classification schemes in use in all natural and social sciences. The main underlying assumption driving a taxonomic class partition is that the entities under study are hierarchically organized: for example, in biology four different species (e.g. lions, tigers, lynxes and cats) can be grouped into two genera (lions and tigers into Pantherinae, lynxes and cats into Felinae), which in turn belong to the same family (Felidae); in social sciences different sub-communities can be grouped in the same community and so on: supporters of different soccer teams can be grouped, at a higher level, as soccer fans that in turn, together with fans of other disciplines, can be grouped as sports fans and so on. Therefore, taxonomic trees carry information about the laws of organization of organisms and communities. In addition, in the last decade much work has been devoted to the study of the statistical features of taxonomic trees, in the hope that they could reveal some general patterns of organization. Interestingly, when looking at the statistical distribution of the sizes of sub-trees (which correspond to the sizes of subspecies within species, of subcommunities within communities) an inverse square power-law relation has been found [1, 2, 3]. Subtree sizes are best viewed by Fig [1 where we show a tree and label every node by the size of its corresponding subtree. It is worth noticing that such scheme corresponds to the statistics of drainage basins area in the river network studies [4]. The presence of a power-law in the size distribution of sub-tree sizes has been hailed as a consequence of some self-organization (possibly critical) of the system under consideration. Actually, and even more interestingly, the robustness of such a law across different taxa and kingdoms (in ecology) and for different social contexts and definitions of community (in social sciences) points to some universal property of these systems.

Here we show that, although self-organization is surely present in ecological and social systems, its presence is not revealed by the above mentioned inverse square relation, which instead is an unescapable consequence of the tree-like nature of classification scheme. Moreover, the robustness of the inverse square relation emerges simply as a universal consequence of the tree-building algorithm.

Community detection within a society is not a simple and perfectly defined task. Indeed, the definition itself of a community is not clear-cut, and even for a given definition the procedure to identify communities might not be easy to implement. Quite recently Girvan and Newman (GN) have proposed an algorithm that, on test cases (where the communities are already known) seems to perform quite effectively [5]. The algorithm relies on a simple intuition: communities are groups of individuals tightly connected with each other, and less connected to the rest of the social network. Therefore, by identifying a suitable parameter that quantifies such degree of connectedness, it is possible to devise a procedure to fragment the social network in communities. GN use the edge betweenness [ 6] index to estimate how much an edge of the network is important to keep it connected: in general edges within a community have a low betweenness value, since the global connectivity is anyway ensured by the large amount of edges linking individuals in the community. Edges between two different communities, on the other hand, should exhibit high betweenness values, since there should be a few of them, sustaining all the social relations between the two communities. The GN algorithm recursively prunes the network by cutting the edge with the largest betweenness, checking whether the network has split in two fragments, and repeating the procedure untill the network is made of disconnected nodes. Any time there is a splitting, the taxonomic tree of communities is updated: the community that has split into two subcommunities gives rise to two offsprings. The algorithm therefore builds a binary tree that encodes the community structure of the social network. More recently, other algorithms [3, 7] have been proposed that run faster than the GN one by relying on local quantities, such as the edge clustering (the computation of the betweenness is costly since it is a global quantity). Yet, the common feature is that the algorithm still produces binary taxonomic trees. As mentioned above, all these algorithms 
correctly identify communities in selected test cases, and all exhibit an inverse square law behavior in the community size distribution, sometimes used as evidence of a self-organized hierarchical structure in the system. Yet, the structure, encoded in a taxonomic tree, is hierarchical by construction, and also the inverse square power behavior is an inescapable outcome of the construction.

It has been analytically shown recently that the subtree size distribution (the community size distribution in the social context) of a random tree is always power-law distributed, $P(s) \sim s^{-\gamma}$, with the exponent $\gamma$ that can be equal to $3 / 2$ in the case of critical random trees [8] (a critical random tree is a tree where nodes have, on the average, just a single offspring), and 2 in the more common case of supercritical trees (the average number of offsprings is larger than one) 9]. To check whether this result applies in general to the detection algorithm, we have applied the one based on the edge clustering coefficient to a simple Erdos-Renyi network made of 3000 nodes and with an average degree equal to 10. As shown in Fig 2 the community size distribution shows an inverse power-law behavior for almost two decades. Yet, the network is completely random, and there is no selforganization whatsoever in the communities identified by the algorithm. The application of the betweenness based GN algorithm gives similar results (data not shown). In Fig 2 we have logarithmically binned the data: this is helpful to improve the quality of the plot, although not essential to reveal the power-law in the community size distribution (the non binned data are just slightly noisier). The cumulated distribution, instead, wouldn't have shown any of these features, as Fig 2 clearly shows: this is a caveat in the use of cumulated distributions, that can sometimes heavily distort the results, to the point that by their use the universality of the inverse square power relation could have been missed.

The inverse square-law behavior also emerges from real data and from sets of real data after some randomization. As an example we examine data from plant taxonomy, which is a particular case of biological classification. We have considered a number of plants ecosystems belonging to different climatic and geographical environments around the world, each one represented by the taxonomic classification tree. For all these trees the study of the statistical distribution of the sizes of subtrees shows a very good inverse power-law distribution with an exponent $\gamma \sim 1.9 \pm 0.2$. As all the plants species in each tree belong to a same ecosystem, this result could be viewed as a sign of some self-organization of the ecosystem itself. Yet, in this case a simple experiment shows that community statistics do not reveal any structure (although the details of the community partition do). We have studied two different kinds of subsets of the above ecosystems: geographical subsets made of groups of species that live toghether in a restricted area [10] and give rise to a small ecosystem, and some other completely random subsets. The statistical distribution of the sizes of subtrees shows an inverse square power-law behavior for both kinds of subsets (see Fig 31). Finding this behavior both in homogeneous data from the same ecosystem, and in a random subsets of species where no correlations are present, shows once more that the inverse square-law is simply a consequence of the tree-like nature of the taxonomic classification. It is worth observing, in this case, that Fig 3 shows the cumulated distribution, that in this case preserves its power-law features (this is further evidence that, if the cumulated distribution is a power-law then the original is power-law too, whereas the inverse is not necessarily true, for example if data are quite noisy).

In conclusion we have shown that the presence of an inverse square-law relation for the distribution of the sizes of communities and species does not mark any significative organization within the system under consideration, but rather it is an inevitable consequence of the classification scheme. More generally we point out that such power-law behavior should be expected whenever the classification scheme used to organize a set of data is based on the construction of a hierarchical tree. This should also apply to classical taxonomy, where the appearance of power-laws in the distributions of subspecies within species has been linked to some intrinsic fractality in the way species are organized. The degree of correlation and organization of the system under study should be instead tied to the quality of the scaling rather than to its bare presence. These subtle relations are yet to be discovered. In some cases, the community size distribution has shown power-law scaling with exponent $3 / 2$, rather then 2[11]: since it is known that the only ingredient necessary to find such a scaling for random trees is their criticality (number of offsprings equal to 1 ), we speculate that it is not the presence of scaling itself that points to some peculiarities in these systems, but rather the specific value of the exponent (and we stress that no other exponents different from 2 and $3 / 2$ could be found).

We thank the EU FET Open project IST-2001-33555 COSIN, the OFES-Bern under contract 02.0234.
[1] B. Burlando, J. Theor. Biol. 14699 (1990).

[2] B. Burlando, J. Theor. Biol. 163 161(1993).

[3] F. Radicchi, C. Castellano, F. Cecconi, V. Loreto, D. Parisi ArXiv:cond-mat/0309488

[4] I. Rodriguez-Iturbe and A. Rinaldo, Fractal River Basins: Chance and Self-Organization Cambridge Univ. Press, (1997).
[5] M. Girvan and M.E.J. Newman, Proc. Natl. Acad. Sci. USA 99, 7821 (2002).

[6] L. Freeman, Sociometry 40, 35 (1977).

[7] M.E.J. Newman and M. Girvan ArXiv:cond-mat/0308217

[8] T.E. Harris, The theory of branching processes. SpringerVerlag, Berlin, (1963). 


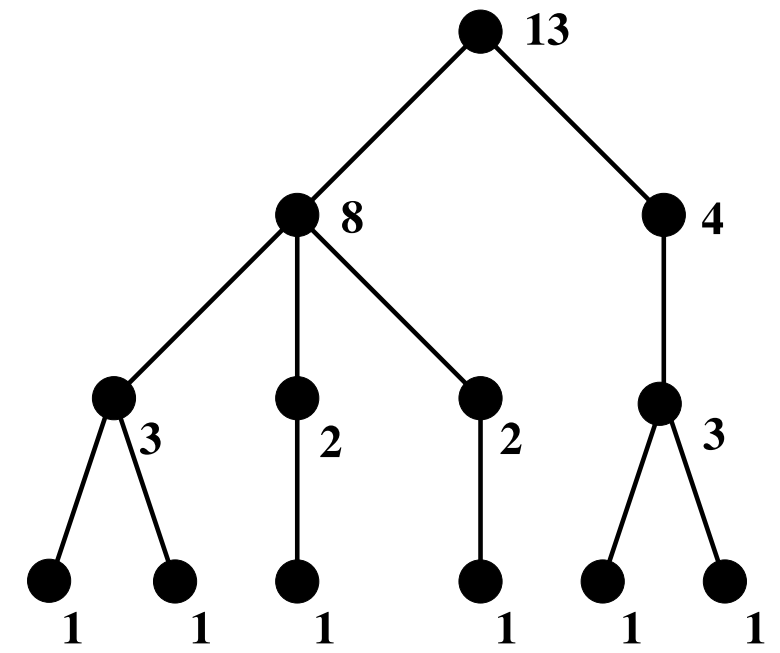

FIG. 1: Binary tree with nodes labelled by their sub-tree sizes.

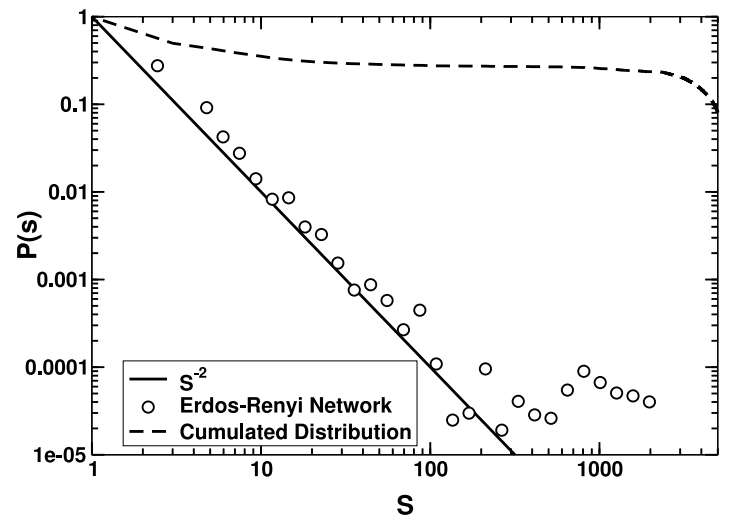

FIG. 2: Community size distribution for an Erdos-Renyi network of 3000 nodes and with average degree equal to 10 . The straight line is $s^{-2}$.
[9] P. De Los Rios Europhys. Lett. 56, 898 (2001)

[10] B. Anzalone, Elenco preliminare delle piante vascolari spontanee del Lazio, Regione Lazio e Società Botanica Italiana, 1984.

[11] R. Guimera, L. Danon, A. Diaz-Guilera, F. Giralt and A. Arenas, ArXiv:cond-mat/0211498 


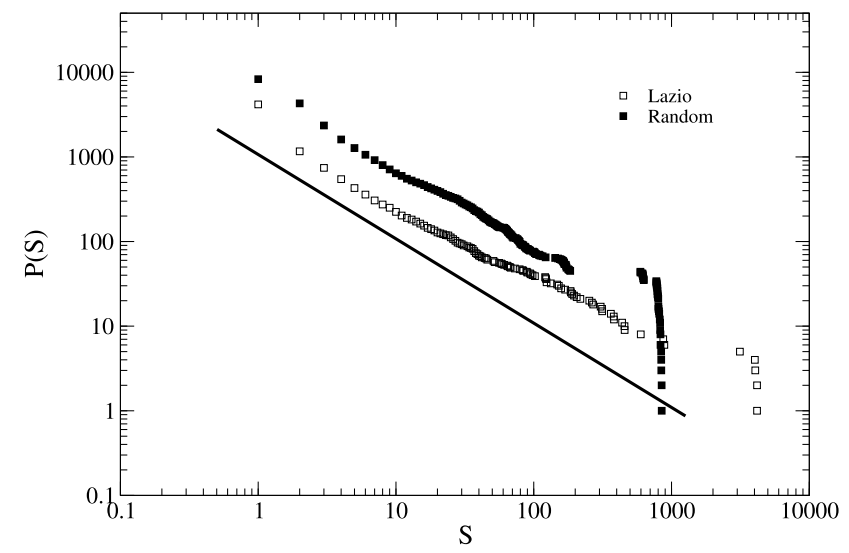

FIG. 3: Community size distribution for the flora of Lazio and a random subset of italian species. The straight line is $s^{-1}$ since these are cumulated distributions. 